\title{
Luxations aiguës d'épaule : évaluation rétrospective aux urgences et proposition d'un protocole de sédation procédurale*
}

\section{Acute Shoulder Dislocation in the Emergency Department: A Retrospective Evaluation of Pain Management and a Proposal for a Standard Operating Procedure}

\author{
O. Guillot · P. Le Borgne $\cdot$ P. Kauffmann $\cdot$ J.P. Steinmetz $\cdot$ M. Schaeffer $\cdot$ C. Kam $\cdot$ C.E. Lavoignet $\cdot$ P. Adam $\cdot$ \\ P. Bilbault
}

Reçu le 23 octobre 2017; accepté le 24 novembre 2017

(C) SFMU et Lavoisier SAS 2017

Résumé Introduction : Les luxations d'épaule sont des pathologies fréquentes aux urgences, parmi les plus douloureuses et pour lesquelles il n'existe pas de recommandations spécifiques. Elles représentent $45 \%$ de l'ensemble des luxa-

O. Guillot · P. Le Borgne $(\bowtie) \cdot$ P. Kauffmann · C. Kam ·

C.E. Lavoignet $\cdot$ P. Bilbault

Service d'accueil des urgences adultes,

hôpitaux universitaires de Strasbourg,

hôpital de Hautepierre, 1 avenue Molière,

67200 Strasbourg, France

e-mail : pierrick_med@yahoo.fr

P. Le Borgne · P. Bilbault

EA 7293, Fédération de médecine translationnelle

de Strasbourg (FMTS), université de Strasbourg,

4 rue Kirschleger,

67000 Strasbourg, France

P. Le Borgne $\cdot$ P. Adam · P. Bilbault

Faculté de médecine, université de Strasbourg,

4 rue Kirschleger, 67000 Strasbourg

J.P. Steinmetz

Service de chirurgie digestive et générale, hôpitaux universitaires de Strasbourg,

hôpital de Hautepierre, 1 avenue Molière,

67098 Strasbourg, France

\section{Schaeffer}

Département de santé publique,

hôpitaux universitaires de Strasbourg,

1 place de l'Hôpital, 67000 Strasbourg, France

P. Adam

Service de chirurgie orthopédique et traumatologique,

hôpitaux universitaires de Strasbourg, hôpital de Hautepierre,

1 avenue Molière, 67200 Strasbourg, France

*Ce travail a fait l'objet d'une communication orale lors du congrès de la Société Française de Médecine d'Urgence (SFMU)

à Paris en juin 2016. tions. L'objectif principal de cette étude était d'analyser la gestion de la douleur dans cette pathologie.

Matériel et méthodes : Nous avons conduit une étude observationnelle, monocentrique et rétrospective colligeant 238 patients ayant consulté aux urgences pour une luxation aiguë d'épaule (01/01/2012-30/04/2015). Les luxations réduites spontanément, les luxations sur prothèse et les luxations chroniques étaient exclues de l'étude.

Résultats : Les luxations aiguës d'épaule étaient majoritairement réduites aux urgences (90\%, IC95\% : [86-94]). Il s'agissait d'une pathologie hyperalgique (Echelle numérique médiane : 8/10, [EIC : 6-9]) touchant plutôt les sujets jeunes (âge médian : 48 ans, EIC : 28-73). Il existait une grande hétérogénéité dans la répartition des antalgiques utilisés, le mélange équimolaire d'oxygène et de protoxyde d'azote (MEOPA) était l'antalgique le plus prescrit (50\% (IC95 \% : [42-55])), suivi par le paracétamol (25\%) et le néfopam (15\%). Dans le sous-groupe des luxations compliquées d'une fracture, le MEOPA était également le plus prescrit. La répartition des autres antalgiques était par ailleurs similaire. Enfin, la comparaison entre les deux équipes médicales (urgentiste vs orthopédiste) ne retrouvait aucune différence de prescription en dehors du midazolam davantage utilisé par les urgentistes $(16 \%$ vs $4 \%, \mathrm{p}=0,001)$.

Conclusion : Il existait une grande hétérogénéité des pratiques et une sous-utilisation d'antalgiques et d'hypnotiques malgré des douleurs importantes dès l'admission. Un chemin clinique visant à améliorer cette prise en charge est proposé afin d'homogénéiser les pratiques.

Mots clés Luxation aiguë d'épaule · Sédation procédurale · Antalgie

Abstract Introduction: Shoulder dislocations are a frequent pathology in the Emergency Department (ED), they are very painful and there are no specific guidelines for their 
management. They represent $45 \%$ of all dislocations. The main objective of this study was to analyze pain management in the ED.

Methods: We conducted a monocentric, observational and retrospective study including 238 patients who presented to the ED with acute shoulder dislocation from January 1, 2012 to April 30, 2015. We excluded spontaneously reduced dislocations, prosthetic dislocations and chronic dislocations. Results: Acute shoulder dislocations were mostly reduced in the $\mathrm{ED}(90 \%, 95 \% \mathrm{CI}:$ [86-94]). It remains an hyperalgic condition (median Visual Analog Scale [VAS] 8/10 [IQR : 6-9]), affecting young patients (median age : 48 years, IQR : 28-73). The Equimolar Mixture of Oxygen and Nitrous Oxide (EMONO) was the most prescribed analgesic for reduction in 50\% of all patients (95\% CI : [42-55]) followed by paracetamol $(25 \%)$ and nefopam $(15 \%)$. Furthermore, in the subgroup of fracture complicated dislocations, EMONO was also the most frequently prescribed analgesic. The distribution of other analgesics was otherwise similar. Finally, the comparison between the two medical teams (emergency physician and orthopedic surgeons) was similar and found only one difference, which concerned midazolam prescription. It was more often used by emergency physicians $(16 \%$ vs $4 \%, \mathrm{p}=0.001$ ).

Conclusion: This study revealed that pain management is often sub-optimal with a very low use of analgesics and hypnotics despite high intake VAS. A standard operating procedure to improve the overall management of these patients will be here proposed.

Keywords Acute shoulder dislocation - Procedural sedation · Pain management

\section{Introduction}

Bien que la douleur représente le principal motif de consultation aux urgences, plusieurs travaux soulignent des difficultés dans la prise en charge antalgique des patients consultant aux urgences pour un motif douloureux [1-3]. L'absence de recours à des protocoles pré-établis et une fréquente sousévaluation de la douleur ressortent comme des facteurs entretenant « l'oligo-analgésie » souvent décrite aux urgences [4,5]. Ainsi, ces mêmes problématiques font débat au sujet de la prise en charge antalgique des luxations aiguës d'épaule amenant à la comparaison des diverses méthodes antalgiques [6]. Les luxations aiguës d'épaule (majoritairement antéromédiales) représentent le type de luxation le plus fréquent $(45 \%$ de l'ensemble des luxations) et le plus douloureux. Ceci représente une incidence d'environ 8/100 000 personnes par an et une prévalence d'environ $2 \%[7,8]$. Le médecin urgentiste sera en première ligne face à ces patients ; en effet le recours à une prise en charge chirurgicale reste rare, réservée le plus souvent aux luxations compliquées d'une fracture articulaire. Malgré la fréquence de cette pathologie, de nombreux éléments de sa prise en charge restent mal codifiés. Parmi les nombreuses problématiques pouvant apparaître au cours de la prise en charge initiale, nous avons choisi de nous pencher sur la question de l'antalgie dans les luxations aiguës d'épaule. En effet, le recours à des antalgiques associés à des agents sédatifs se discute dans cette indication. Le but de cette association est de diminuer la douleur et la contraction musculaire, causée par la pathologie et acutisée par le geste thérapeutique, tout en permettant le maintien d'une ventilation spontanée [9]. Ce furent les objectifs de " la sédation procédurale » des recommandations formalisées d'expert SFAR-SFMU parues en 2012 [10]. Dans ce contexte, en présumant d'une probable hétérogénéité des pratiques en termes de prise en charge antalgique, due notamment à l'absence de recommandations ; l'objectif principal de notre étude était d'analyser la prise en charge de la douleur dans les luxations aiguës d'épaule aux urgences adultes d'un centre hospitalo-universitaire (CHU).

\section{Matériel et méthodes}

Nous avons réalisé une étude rétrospective, observationnelle, monocentrique, au sein du service des urgences d'un CHU français (70 000 passages par an) sur une durée totale de 41 mois (janvier 2012-avril 2015). Tous les patients ayant consulté aux urgences pour une luxation aiguë d'épaule, associée ou non à un autre motif de consultation sur la période de l'étude, étaient inclus, soit un nombre initial de 286 dossiers sélectionnés grâce au codage CIM 10 (Classification internationale des maladies, $10^{\mathrm{e}}$ révision). Les critères d'exclusion étaient : les luxations sur prothèse, les luxations asymptomatiques de découverte fortuite et enfin les patients présentant une luxation déjà réduite à leur arrivée aux urgences. Ainsi, pour chaque patient admis durant la période de l'étude, nous avons exploité les données recueillies à partir des dossiers médicaux informatiques, soit : la feuille d'admission administrative, la fiche d'admission de l'infirmier d'accueil et d'orientation (IAO), l'observation médicale, la feuille de surveillance des constantes, les prescriptions médicamenteuses et les examens radiologiques. Le travail initial a consisté à analyser différents paramètres de la prise en charge médicale et paramédicale. Nous nous attacherons à présenter ici uniquement les résultats ayant trait à la prise en charge antalgique de ces patients. Concernant cette prise en charge, nous avons analysé les critères suivants : l'échelle numérique de la douleur (EN) à l'admission, l'antalgie (médicamenteuse ou non médicamenteuse) mise en œuvre, le nombre d'EN relevées, le nombre de dossiers sans aucune mention de prescription d'antalgique tout au long de la prise en charge, le type d'antalgique prescrit en considérant la liste 
suivante (MEOPA, tramadol, midazolam, antiinflammatoire non stéroïdien, morphine, fentanyl, kétamine, paracétamol, propofol, codéine, néfopam). Nous avons également effectué trois analyses en sous-groupe afin de comparer la prise en charge antalgique des patients présentant une luxation aiguë d'épaule en fonction : de la présence ou non d'une fracture, du lieu de prise en charge (circuit ambulatoire vs circuit conventionnel couché), de l'équipe ayant pris en charge le patient (chirurgien vs urgentiste). En effet, dans notre centre, les chirurgiens orthopédistes ou les médecins urgentistes se répartissaient la gestion des urgences traumatologiques en ayant le même plateau technique à disposition.

L'accord de la Commission Nationale de l'Informatique et des Libertés (CNIL) a été obtenu en vue d'exploiter les données (numéro autorisation 1993010v0). Le protocole de recherche a également été accepté par le Comité d'éthique de la Faculté de médecine de Strasbourg le 29 septembre 2016 (numéro d'autorisation : 2016-59).

\section{Analyse statistique}

L'analyse statistique descriptive des variables quantitatives s'est faite en donnant, pour chaque variable, les paramètres de position (moyenne pour les variables gaussiennes et médiane pour les autres) ainsi que les paramètres de dispersion (écart-type pour les variables gaussiennes et écart interquartile pour les autres). La normalité des données a été testée par le test de Shapiro-Wilk. Pour les variables qualitatives, les pourcentages et les pourcentages cumulés ont été analysés pour chaque variable. Pour l'analyse univariée, l'indépendance des variables a été évaluée par les tests statistiques du $\chi^{2}$, de Fisher et de Mann-Whitney-Wilcoxon, choisis en fonction de la nature des variables. Une relation était considérée significative entre les variables si le $\mathrm{p} \leq 0,05$ en situation bilatérale. L'ensemble de ces données a été colligé sur un tableur Excel 2011. Elles ont été ensuite analysées avec un logiciel d'analyse statistique (logiciel $\mathrm{R}$ dans sa version 3.0.0) en collaboration avec le département de santé publique du CHU.

\section{Résultats}

Sur les 238 patients inclus pour analyse statistique (Fig. 1), $66 \%$ d'entre eux étaient des hommes. L'âge médian des patients était de 48 ans (écart interquartile, EIC : 28-73) avec un minimum de 18 ans et un maximum de 96 ans. Les chutes représentaient la principale cause de luxations (49\%) et $38 \%$ des patients avaient un antécédent de luxation d'épaule. Parmi ces patients, un nouvel épisode survenait dans la moitié des cas du même côté (48\%). Le lieu de la

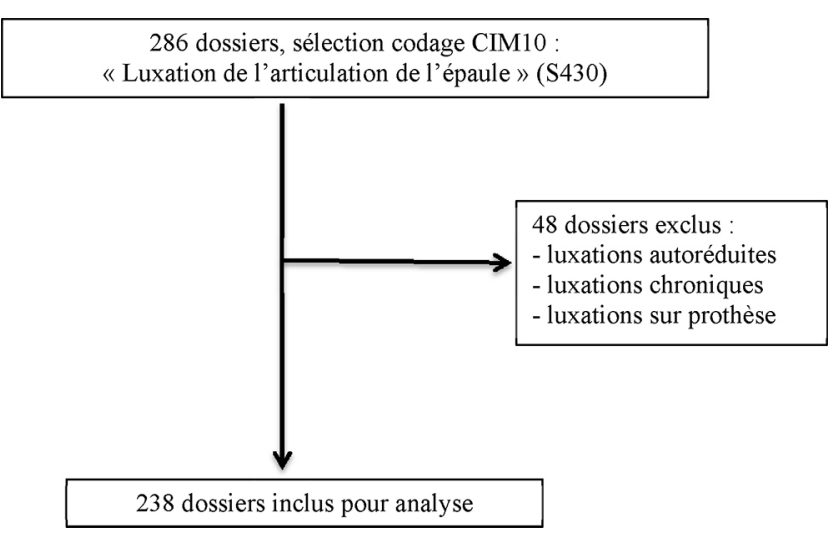

Fig. 1 Diagramme de flux de l'étude. CIM10 : classification internationale des maladies, $10^{\mathrm{e}}$ révision

prise en charge était majoritairement le circuit de traumatologie ambulatoire des urgences (76\%). L'équipe prenant initialement en charge le patient était composée d'urgentistes dans $54 \%$ des cas et de chirurgiens orthopédistes dans le reste des cas (46\%). Le temps médian de prise en charge totale aux urgences était de 153 minutes (IQR : 87$273 \mathrm{~min}$ ). Le délai médian de prise en charge entre l'admission et la réalisation du bilan radiologique pré-réduction était de 20 minutes (IQR : 10-45 min). Le service des urgences était le lieu de la réduction dans la majorité des cas $(90 \%$, IC95 \% : [86-94]), le bloc opératoire sous anesthésie générale l'était dans $8 \%$ des cas et pour $2 \%$ des dossiers, cette information n'était pas clairement identifiable. Les complications les plus fréquemment associées aux luxations étaient les complications fracturaires dans $17 \%$ des cas (IC95 \% : [12-22]) et les complications neurologiques (déficit sensitif ou moteur) retrouvées dans $6 \%$ des cas.

Sur l'ensemble de la population de l'étude, l'EN médiane à l'admission aux urgences était de 8/10 (EIC : 6-9) sur les 178 dossiers $(75 \%)$ où l'information était renseignée. La prise en charge antalgique était instituée dès l'IAO dans seulement $21 \%$ des cas grâce à la mise en place d'un protocole de délégation de prescription. La répartition des différents antalgiques utilisés pour la réduction est résumée dans le Tableau 1, le MEOPA était l'antalgique le plus prescrit (50\%). La médiane d'EN reportée sur toute la durée de prise en charge était de 2 (EIC : 1-3) relevées, ceci correspondait à un relevé d'EN toutes les 87 minutes.

La première analyse en sous-groupe portait sur l'ensemble des patients présentant une luxation aiguë d'épaule compliquée d'une fracture soit 78 patients (Tableau 2). Il ressort de cette comparaison que, même en présence d'une fracture, celle-ci était majoritairement prise en charge aux urgences (83\% (IC95 \% : [73-91]). Aucune différence significative, n'était constatée concernant l'antalgie. Le délai de prise en charge de ces patients aux urgences était toutefois nettement plus élevé (temps médian : 218 vs $131 \mathrm{~min}, \mathrm{p}=0,003$ ). 
Tableau 1 Utilisation des différents antalgiques au cours des manœuvres de réduction des luxations d'épaule sur la population de l'étude

\begin{tabular}{|ll|}
\hline Antalgiques & n (\%) \\
\hline MEOPA & $119(50)$ \\
Paracétamol & $59(25)$ \\
Néfopam & $36(15)$ \\
Tramadol & $26(11)$ \\
Midazolam & $26(11)$ \\
Morphine & $12(5)$ \\
Autres benzodiazépines & $2(1)$ \\
Kétamine & $2(1)$ \\
Propofol & $1(0,5)$ \\
\hline
\end{tabular}

Résultats exprimés en nombre (\%) sur un effectif total de 238 patients. MEOPA : mélange équimolaire d'oxygène et de protoxyde d'azote ; IC $95 \%$ : intervalle de confiance à $95 \%$

La seconde analyse en sous-groupe (en fonction du secteur de prise en charge) a permis de constater ainsi que la prescription de certains antalgiques (paracétamol et tramadol) était sensiblement plus élevée en secteur conventionnel (Tableau 3). Le délai médian de réalisation du bilan radiologique initial était de 30 (19-60) minutes dans le circuit conventionnel vs 6 (5-20) minutes en circuit ambulatoire $(\mathrm{p}<0,001)$. La durée totale de prise en charge était également significativement plus importante en secteur conventionnel couché ( 285 vs 124 minutes, $p<0,001)$.

Enfin, une dernière analyse en sous-groupe a permis de comparer la prise en charge des patients en fonction de l'équipe ayant en charge le secteur traumatologique des urgences adultes (Tableau 4). La prescription d'antalgique était sensiblement comparable entre les deux groupes hormis pour le midazolam prescrit davantage dans le groupe des urgentistes $(16 \%$ vs $4 \%, p=0,001)$. Le délai médian total de prise en charge était par ailleurs significativement plus important dans le groupe urgentistes (190 vs 109 minutes, $\mathrm{p}<0,001)$.

Nous avons également recherché si certaines caractéristiques cliniques connues au moment de la réduction auraient pu orienter le praticien vers le choix d'une classe d'antalgique. Ainsi, nous avons examiné la relation entre plusieurs paramètres : l'équipe et le lieu de prise en charge, la présence d'antécédents de luxation, le mécanisme de la luxation, la présence de fracture associée à la luxation, la réduction aux urgences ou au bloc opératoire, l'EN d'entrée, l'âge du patient et la prescription d'au moins un traitement antalgique, la prescription de MEOPA seul et la prescription de morphine seule. Deux associations sont apparues comme significatives : la relation entre l'âge et la prescription de
Tableau 2 Comparaison de la prise en charge des luxations aiguës d'épaule compliquées ou non d'une fracture

\begin{tabular}{|c|c|c|c|}
\hline & $\begin{array}{l}\text { Luxation } \\
\text { avec fracture } \\
n=78\end{array}$ & $\begin{array}{l}\text { Luxation sans } \\
\text { fracture } \\
n=160\end{array}$ & $\begin{array}{l}\text { Valeur } \\
\text { de } p\end{array}$ \\
\hline \multicolumn{4}{|l|}{ Variables générales } \\
\hline Âge (années) & $51(28-70)$ & $42(28-72)$ & 0,78 \\
\hline $\begin{array}{l}\text { Réduction } \\
\text { aux urgences }\end{array}$ & $64(83)$ & $150(94)$ & 0,48 \\
\hline Réduction au bloc & $14(19)$ & $5(3)$ & 0,001 \\
\hline $\begin{array}{l}\text { Complications } \\
\text { vasculaires }\end{array}$ & $1(1)$ & $0(0)$ & 0,29 \\
\hline $\begin{array}{l}\text { Complications } \\
\text { neurologiques }\end{array}$ & $7(9)$ & $8(5)$ & 0,49 \\
\hline EN à l'IOA & $8(6-10)$ & $7(5-9)$ & 0,07 \\
\hline Nombre d'EN & $2(1-3)$ & $2(1-3)$ & 0,62 \\
\hline \multicolumn{4}{|c|}{ Antalgie pendant réduction } \\
\hline Antalgie à l'IOA & $10(13)$ & $38(24)$ & 0,17 \\
\hline MEOPA & $39(50)$ & $77(48)$ & 0,89 \\
\hline Midazolam & $10(13)$ & $16(10)$ & 0,52 \\
\hline Morphine & $5(6)$ & $8(5)$ & 0,56 \\
\hline Kétamine & $1(1)$ & $2(1)$ & 0,54 \\
\hline Propofol & $0(0)$ & $2(1)$ & 1 \\
\hline Nefopam & $11(14)$ & $24(15)$ & 1 \\
\hline Paracétamol & $24(31)$ & $35(22)$ & 0,11 \\
\hline Tramadol & $14(18)$ & $13(8)$ & 0,03 \\
\hline \multicolumn{4}{|c|}{ Délai de prise en charge (min) } \\
\hline $\begin{array}{l}\text { Admission- } \\
\text { imagerie }\end{array}$ & $20(6-40)$ & $25(10-45)$ & 0,24 \\
\hline Aux urgences & $218(130-344)$ & $131(83-208)$ & 0,003 \\
\hline \multicolumn{4}{|c|}{$\begin{array}{l}\text { Résultats exprimés en nombre (\%) sur un effectif total } \\
\text { de } 238 \text { patients. Médiane (écart interquartile). IOA : infirmier } \\
\text { d'accueil et d'orientation ; MEOPA : mélange équimolaire } \\
\text { d'oxygène et de protoxyde d'azote ; EN : échelle numérique } \\
\text { de la douleur; EIQ : écart interquartile ; med : médiane ; min : } \\
\text { minutes }\end{array}$} \\
\hline
\end{tabular}

MEOPA (davantage prescrit chez les sujets jeunes, $\mathrm{p}=0,04$ ) et la relation entre la prescription d'un morphinique et la présence d'une luxation compliquée d'une fracture (davantage prescrit devant une luxation avec fracture, $\mathrm{p}=0,04$ ).

\section{Discussion}

Les résultats obtenus sur l'ensemble de la population confirment l'intensité de la douleur dans les luxations d'épaule à l'admission (EN médiane : $8 / 10$ ). On note tout d'abord une difficulté à la tracer (réévaluation peu fréquente) et aucune retranscription dans près d'un quart des dossiers $(23 \%)$. La 


\begin{tabular}{|c|c|c|c|}
\hline & $\begin{array}{l}\text { Circuit } \\
\text { Conventionnel } \\
n=43\end{array}$ & $\begin{array}{l}\text { Circuit } \\
\text { ambulatoire } \\
n=195\end{array}$ & $\begin{array}{l}\text { Valeur } \\
\text { de } p\end{array}$ \\
\hline \multicolumn{4}{|c|}{ Variables générales } \\
\hline $\begin{array}{l}\text { Réduction } \\
\text { aux urgences }\end{array}$ & $36(84)$ & $179(92)$ & 0,14 \\
\hline $\begin{array}{l}\text { Réduction } \\
\text { au bloc }\end{array}$ & $7(17)$ & $10(5)$ & 0,01 \\
\hline Nombre d'EN & $2(1-3)$ & $2(1-3)$ & 0,01 \\
\hline EN à l'IOA & $7(5-9)$ & $8(5-9)$ & 0,61 \\
\hline \multicolumn{4}{|l|}{ Antalgie } \\
\hline Antalgie & $5(12)$ & $46(24)$ & 0,1 \\
\hline \multicolumn{4}{|l|}{ à l'IOA } \\
\hline MEOPA & $16(39)$ & $99(51)$ & 0,13 \\
\hline Midazolam & $5(12)$ & $19(10)$ & 0,63 \\
\hline Morphine & $3(7)$ & $8(4)$ & 0,28 \\
\hline Kétamine & $1(2)$ & $1(0,5)$ & 0,41 \\
\hline Propofol & $1(2)$ & $0(0)$ & 0,23 \\
\hline Nefopam & $6(14)$ & $27(14)$ & 1 \\
\hline Paracétamol & $17(41)$ & $39(20)$ & 0,002 \\
\hline Tramadol & $13(30)$ & $12(6)$ & $<0,001$ \\
\hline \multicolumn{4}{|c|}{ Délai de prise en charge (min) } \\
\hline $\begin{array}{l}\text { Admission- } \\
\text { imagerie }\end{array}$ & $30(19-60)$ & $6(5-20)$ & $<0,001$ \\
\hline Aux urgences & $285(183-420)$ & $124(81-192)$ & $<0,001$ \\
\hline \multicolumn{4}{|c|}{$\begin{array}{l}\text { Résultats exprimés en nombre (\%) sur un effectif total } \\
\text { de } 238 \text { patients. Médiane (écart interquartile). IOA : infirmier } \\
\text { d'accueil et d'orientation ; MEOPA : mélange équimolaire } \\
\text { d'oxygène et de protoxyde d'azote ; EN : échelle numérique } \\
\text { de la douleur ; EIQ : écart interquartile ; med : médiane ; min : } \\
\text { minutes }\end{array}$} \\
\hline
\end{tabular}

délégation de prescription d'antalgique (pallier 1 per os), mise en place afin de minimiser le délais d'attente pour les patients hyperalgiques à l'admission était effective dans seulement un quart des dossiers. La majorité des prescripteurs (urgentistes et chirurgiens) choisissaient le MEOPA en première intention lors des manœuvres de réduction. Ces résultats semblent cohérents du fait de la sécurité d'utilisation de ce produit et de sa facilité d'utilisation (économisant notamment la pose d'une voie veineuse). Sur les dix dernières années, de nombreux travaux rapportent l'utilisation du protoxyde d'azote, son efficacité et sa sécurité pour une sédation en urgence chez l'adulte [11-13]. L'analyse univariée a par ailleurs retrouvé un lien entre la prescription de MEOPA plutôt prescrit chez les patients jeunes en comparaison aux autres antalgiques. Ce qui semble également cohérent, puis-
Tableau 4 Comparaison de la prise en charge des luxations aiguës d'épaule en fonction de l'équipe (chirurgiens vs urgentistes)

\begin{tabular}{|c|c|c|c|}
\hline & $\begin{array}{l}\text { Urgentistes } \\
n=129\end{array}$ & $\begin{array}{l}\text { Chirurgiens } \\
n=109\end{array}$ & Valeur de $\mathbf{p}$ \\
\hline \multicolumn{4}{|c|}{ Variables générales } \\
\hline $\begin{array}{l}\text { Réduction } \\
\text { aux urgences }\end{array}$ & $115(90)$ & $98(90)$ & 0,46 \\
\hline $\begin{array}{l}\text { Réduction } \\
\text { au bloc }\end{array}$ & $9(7)$ & $10(9)$ & 0,46 \\
\hline Nombre d'EN & $2(1-3)$ & $2(1-3)$ & 0,78 \\
\hline \multicolumn{4}{|c|}{ Antalgie pendant réduction } \\
\hline MEOPA & $61(48)$ & $53(49)$ & 1 \\
\hline Midazolam & $20(16)$ & $4(4)$ & 0,001 \\
\hline Morphine & $5(4)$ & $6(6)$ & 0,38 \\
\hline Kétamine & $0(0)$ & $2(2)$ & 0,20 \\
\hline Propofol & $0(0)$ & $1(1)$ & 0,45 \\
\hline Nefopam & $24(19)$ & $11(10)$ & 0,07 \\
\hline Paracétamol & $34(27)$ & $24(22)$ & 0,37 \\
\hline Tramadol & $14(11)$ & $13(12)$ & 0,07 \\
\hline \multicolumn{4}{|c|}{ Délai de prise en charge (min) } \\
\hline $\begin{array}{l}\text { Admission- } \\
\text { imagerie }\end{array}$ & $25(10-45)$ & $20(10-45)$ & 0,42 \\
\hline Aux urgences & $190(118-318)$ & $109(65-202)$ & $<0,001$ \\
\hline \multicolumn{4}{|c|}{$\begin{array}{l}\text { Résultats exprimés en nombre }(\%) \text { sur un effectif total } \\
\text { de } 238 \text { patients. Médiane (écart interquartile). MEOPA : } \\
\text { mélange équimolaire d'oxygène et de protoxyde d'azote ; EN : } \\
\text { échelle numérique de la douleur ; EIQ : écart interquartile ; } \\
\text { med : médiane }\end{array}$} \\
\hline
\end{tabular}

qu'un échec de ce procédé est fréquemment constaté chez des patients âgés, ayant des difficultés à inhaler ce mélange gazeux. La prescription de midazolam, retrouvée dans seulement $11 \%$ des dossiers, peut être interprétée comme un recours à une technique d'analgésie-sédation. Mais il semblerait que cette prescription corresponde plutôt à une démarche davantage « prescripteur-dépendant » si l'on en croit la faible quantité des dossiers dans laquelle il apparaît.

Dans l'analyse en sous-groupe comparant les luxations compliquées d'une fracture et les luxations simples, il apparaît que la grande majorité des luxations compliquées de fractures « minimes » (autorisant les manœuvres de réduction) sont prises en charge en quasi-totalité aux urgences sans recours au bloc opératoire. Ce résultat apparaît comme un argument supplémentaire pour parfaire une stratégie de sédation procédurale aux urgences. On peut également voir dans cette absence de consensus concernant l'alternative médicamenteuse à adopter en cas d'échec du MEOPA, un besoin de mettre en place un protocole de prescription d'antalgie adaptée. Nous proposons ainsi un support à la 
prescription sous la forme d'un chemin clinique conçu après discussion avec les équipes de chirurgie orthopédique, les urgentistes et les anesthésistes intervenant conjointement autour de ces patients ainsi que de l'analyse de la littérature en matière de sédation procédurale (Annexe 1). Le but était de proposer une alternative au MEOPA en cas d'échec ou pour les patients les plus difficiles à réduire du fait, souvent, d'une contraction musculaire intense. En effet, la myorelaxation reste une des clés de la réussite d'une réduction. C'est dans cette double logique de soulager et de relaxer que nous avons choisi dans notre protocole l'association midazolam+kétamine $(0,03 \mathrm{mg} / \mathrm{kg}$ pour le midazolam et $0,3-0,5 \mathrm{mg} / \mathrm{kg}$ pour la kétamine). Cette association a l'avantage d'apparaître dans les recommandations formalisées d'experts (2010) dans le chapitre dédié à l'analgésie pour les actes douloureux : « il convient de l'administrer si possible dans un endroit calme, chez un patient prévenu et en utilisant volontiers de faibles doses de midazolam titrées » [10]. Cette même idée est reprise dans une sous-partie traitant des propriétés pharmacologiques des analgésiques utilisables en urgence. Il n'est en revanche question nulle part des doses précises de midazolam à utiliser dans cette association. L'analyse de la littérature au sujet de cette association a permis d'isoler une première série d'article portant sur les phénomènes hallucinatoires de l'association midazolamkétamine lors de la phase de réveil. L'apport du midazolam potentialisant la réduction des effets dysphoriques de la kétamine est évoqué en objectif secondaire de deux études publiées en 2008 (une cohorte prospective de 92 patients dans un service d'urgence et une méta-analyse de 87 articles montrant la sécurité d'utilisation de la kétamine par la faible incidence d'effets cardiorespiratoires graves pendant son utilisation) $[14,15]$. D'autres travaux récents, dont l'objectif principal était de comparer les effets de l'ajout d'une faible dose de midazolam $(0,05 \mathrm{mg} / \mathrm{kg})$ à une dose de kétamine allant juqu'à $1,5 \mathrm{mg} / \mathrm{kg}$, montraient une franche amélioration de la qualité du réveil avec moins de phénomènes d'agitation sans noter d'augmentation d'autres effets secondaires [1619]. Ainsi, dans la cohorte la plus importante de Sener et al. [18] (182 patients), l'adjonction de $0,003 \mathrm{mg} / \mathrm{kg}$ de midazolam à une dose de kétamine de $1,5 \mathrm{mg} / \mathrm{kg}$ IV ou $4 \mathrm{mg} / \mathrm{kg}$ IM (vs l'association kétamine+ placebo) permettait une diminution des phénomènes d'agitation de $17 \%$. Par ailleurs, deux autres études ont retrouvé, en plus des effets bénéfiques du midazolam sur la phase de réveil, l'efficacité analgésique de l'association « kétamine+midazolam » en la comparant à de la morphine seule ou à l'association « midazolam-fentanyl »: les résultats montrent, une nouvelle fois, la diminution des effets secondaires psychodysleptiques avec le midazolam et également une efficacité comparable à l'association morphine seule ou morphine +midazolam [20,21]. L'étude de Chudnofsky et al. [22] montrait également des résultats en faveur de la sécurité d'utilisation de l'association midazolam-kétamine (avec des doses supérieures en kétamine : $2 \mathrm{mg} / \mathrm{kg}$ ). Ainsi, dans sa cohorte de 77 patients, il ne retrouvait aucun effet secondaire psychodysleptique invalidant et seulement $6 \%$ d'effets secondaires respiratoires (apnée ou laryngospasme réversibles d'une durée maximale de 120 secondes). Enfin, l'étude de Tawfic et al. [23], même si elle portait sur l'administration de dose continue de kétamine et de midazolam, se prononcait également en faveur de la sécurité d'utilisation de cette association. Enfin, sur le plan pharmacologique, la kétamine présente quelques avantages non négligeables (en urgence) à savoir une action rapide ( 1 minute) et une demi-vie d'élimination courte $(3 \mathrm{~h})$. Le midazolam présente également les mêmes caractéristiques pharmacologiques (action courte : 2 minutes, et demi-vie d'élimination courte : $1,5 \mathrm{~h}$ ). Suite à l'apport de ces différents travaux et à l'analyse de nos pratiques, nous avons pu concevoir un chemin clinique, celui-ci ne détaillant toutefois que la partie relative à l'antalgie (Annexe 1).

Cette étude comporte également quelques limites, le principal biais est son caractère rétrospectif avec également un nombre important de dossiers informatiques incomplets. Elle est par ailleurs monocentrique ce qui induit de facto un effet centre. Ce biais est non négligeable dans le domaine de l'analgésie-sédation. En effet, en l'absence de recommandations claires dans ce domaine, beaucoup de prescriptions d'antalgique dans cette indication sont probablement 《 service-dépendant » voire «praticien-dépendant ». Il conviendrait donc idéalement de concevoir une étude prospective et multicentrique afin de confronter nos pratiques locales à celles des autres services d'urgences ; mais également un autre travail (avant-après) permettant ainsi de tester la pertinence du protocole proposé.

\section{Conclusion}

La prise en charge antalgique des luxations aiguës d'épaule reste encore mal codifiée et il semble exister une grande hétérogénéité des pratiques dans la gestion de la douleur. Celle-ci est encore sous-optimale, le recours à une association (analgésique+hypnotique) médicamenteuse dans le cadre d'une sédation procédurale (définie dans les recommandations SFMU-SFAR, 2012) devrait être préféré lors des manœuvres de réduction. Dans ce contexte, nous avons conçu un chemin clinique ayant pour principal objectif de guider le clinicien et d'améliorer l'antalgie des patients les plus difficiles à réduire (notamment ceux résistant au MEOPA), chez qui le recours à la réduction au bloc opératoire doit rester exceptionnel.

Liens d'intérêts : Les auteurs ne déclarent aucun lien d'intérêt. 


\section{Annexe 1}

Proposition d'un chemin clinique dans la prise en charge des luxations aiguë d'épaule. IDE : infirmier diplômé d'État ; VVP : voie veineuse périphérique ; EN : échelle numérique ; MEOPA : mélange équimolaire d'oxygène et de protoxyde d'azote ; TC : traumatisme crânien ; ATCD : antécédent ; SAU : service d'accueil des urgences ; AG : anesthésie générale ; HTIC : hypertension intracrânienne ; OH aiguë : alcoolisation aiguë ; CI : contre-indication ; min : minutes ; EIQ : écart interquartile ; med : médiane

\section{CHEMIN CLINIQUE : LUXATION AIGUË D'ÉPAULE}

\section{(1) Évaluation Poste IOA (Infirmier d'Accueil et d'Orientation) : 3 objectifs}

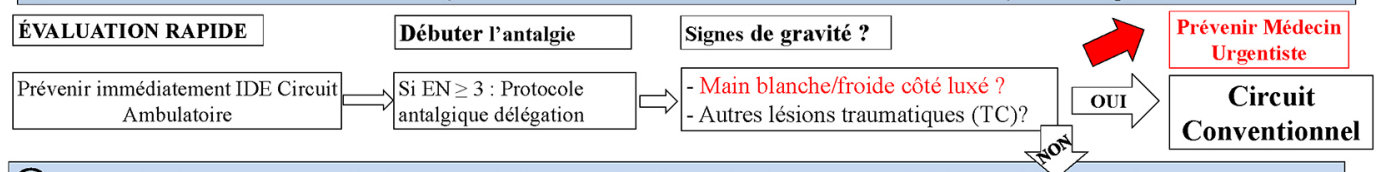

(2) Installation immédiate en Circuit Ambulatoire pour BILAN INITIAL

\begin{tabular}{l|}
\multicolumn{1}{c|}{ Rôle IDE } \\
\hline - Au calme avec à disposition: O2/MEOPA/scope BAVU/ Aspiration \\
- Possibilité pose rapide d'une VVP \\
- Déshabillage patient et installation la plus confortable possible \\
en abduction passive \\
\hline $\begin{array}{l}\text { Consignes de surveillance du patient sous: } \\
\text {-MEOPA : EVA } \\
\text {-Midazolam et kétamine : scopé en circuit conventionnel }\end{array}$ \\
\hline
\end{tabular}

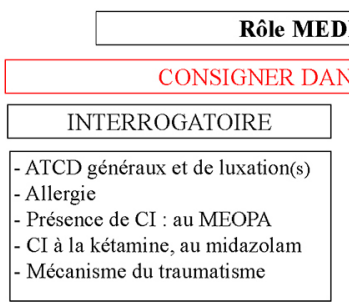

ANS LE DOSSIFR

\begin{tabular}{|l|}
\hline \multicolumn{1}{|c|}{ EXAMEN CLINIQUE } \\
\hline - Déformation? \\
- Complications ? \\
- PEAU : plaie articulaire ? \\
- POULS : pouls radial perçu? \\
- NERF : testing nerf axillaire \\
sensibilité + contractilité deltoíde \\
+ Plexus brachial ? \\
- Autre trauma associé ? \\
\hline
\end{tabular}

(3) Bilan Radio PRÉ RÉDUCTION : clichés épaule Face + Bloom-Obata

\section{(4) Quels types de luxations réduire aux urgences ?}

\begin{tabular}{|l|}
\hline CAS 1 : manœuvres de réduction possible au SAU \\
\hline - Luxation antérieure sans fracture \\
- Luxation antérieure associée à des lésions « de passage » peu \\
importantes conséquence du choc entre la glène et la tête humérale: \\
\hline - encoche superficielle de la tête humérale \\
- image en pan coupé de la glène \\
- ou lésion operculaire du tubercule majeur
\end{tabular}

\begin{tabular}{|l|}
\hline \multicolumn{1}{c|}{ CAS 2 : réduction à prévoir sous AG } \\
\hline - Luxation antérieure associée à des fractures plus importantes (par \\
impaction): \\
- fracture de la tête humérale \\
- fracture du tubercule majeur ou de la glène \\
- autre fracture de l'humérus \\
- Luxation postérieure et erecta
\end{tabular}

\section{(5) Réduction et Antalgie}

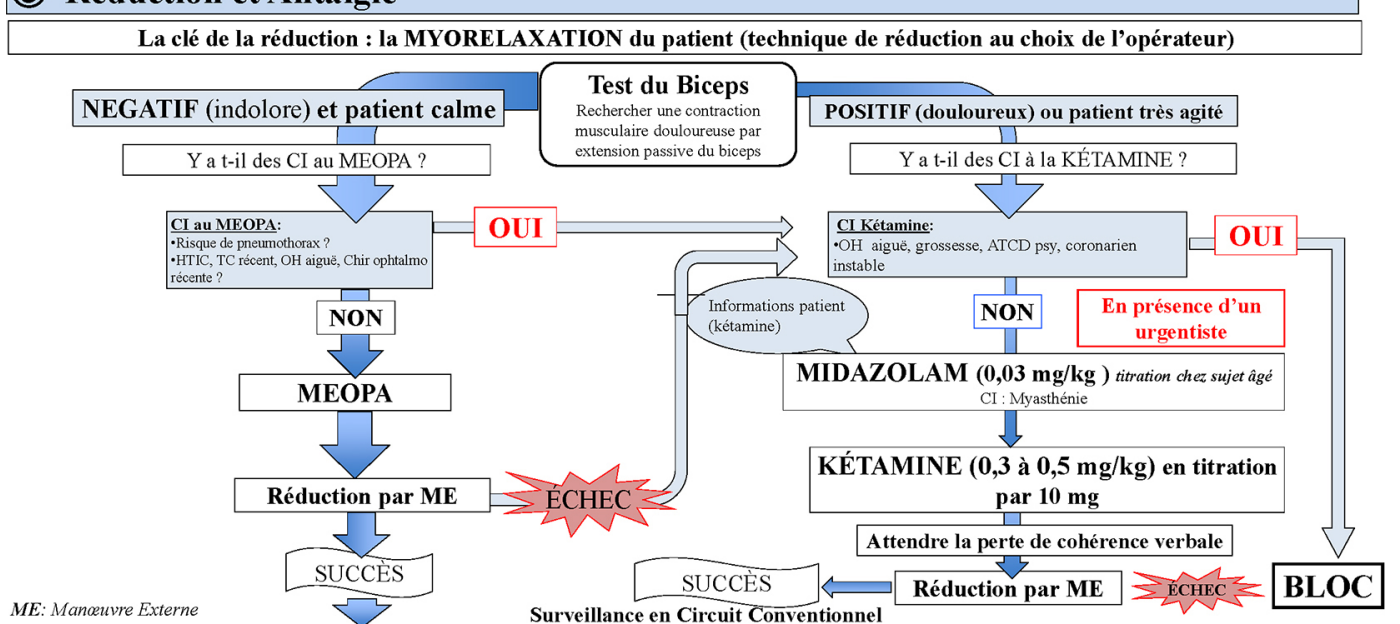

\section{6) Bilan radio POST RÉDUCTION : clichés épaule Face + Bloom-Obata}

\section{(7) AVANT la SORTIE}

- Répéter l'examen clinique de l'épaule (consigner dans le dossier) - Immobilisation en rotation interne coude au corps

- Consultation orthopédique de contrôle à $\mathbf{J} 7$

- Arrêt de travail ? Accident de travail ?

- Personnes âgées seules : autonomie?
Aptitude à la sortie: Score de CHUNG

Évalué à 30 minutes - sortie autorisée si score $\geq 9$ 


\section{Références}

1. Todd KH, Sloan EP, Chen C, et al (2002) Survey of pain etiology, management practices and patient satisfaction in two urban emergency departments. CJEM 4:252-6

2. Todd KH, Ducharme J, Choiniere M, et al (2007) Pain in the emergency department: results of the pain and emergency medicine initiative (PEMI) multicenter study. J Pain 8:460-6

3. Fosnocht DE, Swanson ER, Barton ED (2005) Changing attitudes about pain and pain control in emergency medicine. Emerg Med Clin North Am 23:297-306

4. Ricard-Hibon A, Bounes V, groupe d'experts SFAR-SFMU (2012) Sédation et analgésie en structure d'urgence. Quelles sédation et analgésie chez le patient en ventilation spontannée en structure d'urgence ? Ann Fr Anesth Reanim 31:295-312

5. Carter D, Sendziuk P, Eliott JA, et al (2016) Why is pain Still under-treated in the emergency department? Two new hypotheses. Bioethics 30:195-202

6. Hendey GW (2016) Managing anterior shoulder dislocation. Ann Emerg Med 67:76-80

7. Zacchilli MA, Owens BD (2010) Epidemiology of shoulder dislocations presenting to emergency departments in the United States. J Bone Joint Surg Am 92:542-9

8. Khiami F, Gérometta A, Loriaut P (2015) Management of recent first-time anterior shoulder dislocations. Orthop Traumatol Surg Res 101:S51-57

9. Lachance PA (2012) Reduction of shoulder dislocation: are communication and adequate relaxation more important than technique? Can Fam Physician 58:1189-90, e613-614

10. Bounes V, groupe d'experts SFAR-SFMU (2012) Sédation et analgésie en structure d'urgence. Quelles sont les modalités de réalisation d'une sédation et/ou d'une analgésie pour des actes douloureux. Ann Fr Anesth Reanim 31:340-2

11. Marsden AK (1979) Entonox in the emergency department. Injury 10:311-2

12. Herres J, Chudnofsky CR, Manur R, et al (2016) The use of inhaled nitrous oxide for analgesia in adult ED patients: a pilot study. Am J Emerg Med 34:269-73
13. Gerhardt RT, King KM, Wiegert RS (2001) Inhaled nitrous oxide vs placebo as an analgesic and anxiolytic adjunct to peripheral intravenous cannulation. Am J Emerg Med 19:492-4

14. Newton A, Fitton L (2008) Intravenous ketamine for adult procedural sedation in the emergency department: a prospective cohort study. Emerg Med J 25:498-501

15. Strayer RJ, Nelson LS (2008) Adverse events associated with ketamine for procedural sedation in adults. Am J Emerg Med 26:985-1028

16. Perumal DK, Adhimoolam M, Selvaraj N, et al (2015) Midazolam premedication for Ketamine-induced emergence phenomenon: A prospective observational study. J Res Pharm Pract 4:89-93

17. Somashekara SC, Govindadas D, Devashankaraiah G, et al (2010) Midazolam premedication in attenuating ketamine psychic sequelae. J Basic Clin Pharm 1:209-13

18. Sener S, Eken C, Schultz CH, et al (2011) Ketamine with and without midazolam for emergency department sedation in adults: a randomized controlled trial. Ann Emerg Med 57:109-114.e2

19. Galinski M, Dolveck F, Combes X, et al (2007) Management of severe acute pain in emergency settings: ketamine reduces morphine consumption. Am J Emerg Med 25:385-90

20. Ahmadi O, Isfahani MN, Feizi A (2014) Comparing low-dose intravenous ketamine-midazolam with intravenous morphine with respect to pain control in patients with closed limb fracture. J Res Med Sci 19:502-8

21. Cevik E, Bilgic S, Kilic E, et al (2013) Comparison of ketaminelow-dose midozolam with midazolam-fentanyl for orthopedic emergencies: a double-blind randomized trial. Am J Emerg Med $31: 108-13$

22. Chudnofsky CR, Weber JE, Stoyanoff PJ, et al (2000) A combination of midazolam and ketamine for procedural sedation and analgesia in adult emergency department patients. Acad Emerg Med 7:228-35

23. Tawfic QA, Faris AS, Kausalya R (2014) The role of a low-dose ketamine-midazolam regimen in the management of severe painful crisis in patients with sickle cell disease. J Pain Symptom Manage 47:334-40 